\title{
Wake-up receiver for radio-on-demand wireless LANs
}

Suhua Tang ${ }^{1 *}$, Hiroyuki Yomo ${ }^{2}$, Yoshihisa Kondo ${ }^{1}$ and Sadao Obana ${ }^{1}$

\begin{abstract}
Recent investigations show that access points (APs) of wireless local area networks (WLANs) are idle during much of the time and that an AP in its idle state still consumes a large percentage of power. Wake-up receivers can be used to realize radio-on-demand WLANs, activating APs from the sleep mode only in times of active data communications. A wake-up receiver, sharing the antenna (and the same ISM band) with its co-located WLAN module and exploiting RF energy detection, can be implemented at low cost and run with low power consumption. In this article, we evaluate the effect of an imperfect RF band pass filter (BPF), and suggest a new soft decision method to (i) resist adjacent channel interference leaked by BPF, and, (ii) distinguish wake-up signals from WLAN signals. Extensive simulation and testbed experimental results confirm that the proposed scheme, at a moderate cost, has good performance in delivering wake-up signals and controlling false wake-up events caused by WLAN signals.
\end{abstract}

Keywords: green communications, radio-on-demand, wireless LANs, wake-up receivers, signal recognition

\section{Introduction}

Recent years have seen fast growth of wireless local area networks (WLANs) market. Shipment of WLAN chipsets approaches 1 billion in 2010 [1], among which a large percentage is used in access points (APs). APs, with which mobile nodes associate, extend the Internet backbone. They are always powered on, even in the long idle period, in order to serve potential nodes promptly.

With the trend of global warming, green communications are becoming necessary. Currently, the focus is on how to reduce the huge power consumption due to devices such as routers and switches of the Internet backbone [2] and base stations of cellular networks, where billions of watts are consumed. Although WLAN APs are also a part of the network infrastructure, their power consumption is not taken seriously yet because a single AP merely consumes a few watts [3]. However, there are a large number of APs and all APs in total do consume a huge amount of power. On the other hand, investigations show that some WLAN APs are idle during most of the time [4]. Therefore, it is both necessary

\footnotetext{
* Correspondence: shtang@atr.jp

${ }^{1}$ ATR Adaptive Communications Research Laboratories, 2-2-2 Hikaridai, Seikacho, Soraku-gun, Kyoto 619-0288, Japan

Full list of author information is available at the end of the article
}

and feasible to reduce power consumption of WLAN APs.

The most effective method to reducing power consumption is to put an idle device into sleep. However, a device in sleep is actually separated from the whole network. To make a device in sleep remain connected in the network, either active or passive wake-up schemes are necessary. Schemes suggested for sensor networks, such as CPU driven duty-cycling [5] and wake-up receivers [6-10], can be extended to reduce power consumption of WLAN APs as well. This, however, is non-trivial since WLANs have quite different traffic pattern and application scenarios from sensor networks.

Some prior works suggested wake-up receivers for mobile nodes. A secondary low-power radio, "MiniBrick", is used for wake-up signaling in [11]. But the media access control (MAC) protocol for MiniBricks is left untouched. A low-power sensor mote (802.15.4), working on the same $2.4 \mathrm{GHz}$ industrial, scientific and medical (ISM) band as WLAN modules, is used to monitor WLAN channels in [3]. Since only energy is detected without ID matching, false wake-up probability is high. This is refined in [12], where Zigbee devices are used for more detailed signaling between nodes and APs, to achieve ubiquitous connectivity, high energy 
efficiency and real time handover. As for ID matching, the work in [13] suggests using a bloom filter to match a group of IDs.

Sleep scheduling of WLAN APs is heavily affected by the traffic pattern. (1) In the micro time scale, dutycycling of APs is suggested in [14] for power-efficient multi-hop extension of access networks. Packets can also be aggregated so as to use the burst transmission and high rate of 802.11n [15], enabling a longer idle period and sleeping time. Practically, only the WLAN module can be put into sleep in the short idle period. (2) In the macro time scale, the whole system of idle APs can be put into sleep to realize green WLANs [4]. A further optimization is to aggregate flows to few APs and put more APs into sleep. But some APs still have to stay awake even in the idle state in order to ensure coverage. Several methods may be used to activate APs from sleep. An AP is composed of WLAN module, LAN module, CPU, memory, hard disk etc. A simple wake-up policy is to keep the LAN module and WLAN module awake while putting other modules into sleep, and exploit the wake-on-LAN [16] and wake-on-WLAN [17] functions for wake-up signaling. According to [3], although an idle LAN module consumes little power, an idle WLAN module still consumes significant power (on the order of $1 \mathrm{~W}$ ) in monitoring the channel. A more energy-efficient method is to put the WLAN module into sleep as well, and use an auxiliary low-power (no more than $1 \mathrm{~mW}$ ) wake-up receiver to trigger the wakeup event instead. Currently little work is done on this topic for WLAN APs.

In our previous work, we suggested using wake-up receivers to realize radio-on-demand (ROD) WLANs in the $2.4 \mathrm{GHz}$ ISM band [18], aiming at reducing power consumption of APs due to macro time scale idleness. A wake-up ID (WID) is transmitted by on-off keying (OOK). A wake-up receiver extracts the wake-up signal by a radio-frequency (RF) band pass filter (BPF) and recovers the WID by non-coherent envelope detection. Coexistence of wake-up signals and WLAN signals and recognition of wake-up signals from WLAN signals are studied.

In this article, we further evaluate the effect of adjacent channel interference caused by an imperfect RF BPF. Soft decision (SoftDec) instead of hard decision (HardDec) is suggested. Meanwhile, signal recognition capability is retained. The effect of quantization is also studied. We contribute in two-fold compared with [18]:

- The method to detecting wake-up signals in [18] is based on the assumption that a BPF can completely remove out-of-band interference. However, channels used by WLAN devices are no longer orthogonal when an imperfect BPF is used to extract wake-up signals. A new SoftDec method is suggested for detecting wake-up signals, by mitigating adjacent channel interference leaked due to the imperfect BPF.

- Signal recognition in [18] is limited to HardDec. Furthermore, plain SoftDec, based on correlation, has no capability in distinguishing signals. Proper parameters are suggested for the SoftDec to divide signal space and distinguish wake-up signals from WLAN signals.

Extensive simulation and testbed experiments confirm that the proposed scheme has good performance in delivering wake-up signals and controlling false wake-up events caused by WLAN signals, even in the presence of interference.

The rest of this article is organized as follows: Section 2 discusses design issues of wake-up receivers. Section 3 first presents the system model. Then, processing of wake-up signals and control of false wake-up probability are described in detail. Evaluation results are presented and analyzed in Section 4. Finally Section 5 concludes the article and points out future work.

\section{Design issues of ROD WLANs}

Wireless local area networks APs, as a part of network infrastructure, are turned on all the time, despite the fact that many APs are idle during most of the time. A ROD WLAN is aimed to provide radio access service in an on-demand manner, reducing power consumption of APs meanwhile ensuring a low access delay. In this section, we discuss design issues of wake-up transceivers for ROD WLANs by taking a comparison with sensor networks. Following Table 1 which summarizes the comparison, we first analyze the traffic pattern of WLAN and the available wake-up policy in Section 2.1. Then, we explain the reason for sharing antenna and channel between wake-up transceivers and WLAN devices in Section 2.2 and describe the tradeoff between simplicity and reliability in designing the wake-up receiver in Section 2.3. We also show the budget of power consumption for wake-up receivers in Section 2.4. Finally, we briefly summarize the design freedom and challenges in Section 2.5.

Table 1 Comparison of sensor networks and ROD WLANs

\begin{tabular}{lll}
\hline & Sensor networks & ROD WLANs \\
\hline Duty cycle & Micro time scale & Macro time scale \\
Wake-up latency & Low & Large \\
Antenna/channel & Separate & Shared \\
Signal coexistence & Seldom a concern & Necessary \\
\hline
\end{tabular}




\subsection{Traffic pattern and wake-up policy}

Traffic pattern heavily affects sleep scheduling and the wake-up policy is limited by the boot time of devices. Sensor networks have low duty cycle where packets are generated occasionally. Sensor nodes, using special embedded system, can wake up with a low latency. Therefore, either duty cycling or wake-up receivers can be used. The micro time scale idleness can be effectively exploited and sensor nodes can sleep between two successive transmissions.

As for WLANs, traffic volume varies with time. In the micro time scale, even when an AP is transmitting packets, there may be short idle periods, during which a WLAN module can be temporarily put into sleep. A WLAN AP uses general OS to process data and management frames. Wake-up of the whole system of an AP (including CPU, memory, and hard disk etc. besides the WLAN module) would take a relatively long time, usually on the order of seconds. Hence, it is not feasible to turn off the whole system of an AP to exploit the short idleness. For the same reason, the duty cycling scheme, which will consume significant power in the periodical sleep/wake-up of the whole system, is not proper for WLAN APs. Therefore, active wake-up receivers are used in this design. It only causes initial wakeup latency, which is still endurable. Interested readers may refer to [19] for other types of wake-up receivers.

We aim at reducing power consumption of WLAN APs due to the macro time scale idleness. In the macro time scale, most APs in offices are idle in the night while many APs at home are idle in the daytime. In the proposed scheme, all idle APs are put into sleep compared with [4], where some idle APs are kept awake to ensure coverage. Duty cycling control of a WLAN module in the micro time scale [15] can be exploited in our ROD WLANs as a supplement.

\subsection{Sharing antenna and channel}

Most previous works use a separate channel $(900 \mathrm{MHz}$ [7]) for wake-up signals, and require an extra antenna besides the one used by data communication module. As a comparison, in the ROD WLANs, a wake-up transceiver shares antenna with the co-located WLAN module to reduce the hardware cost. As a result, the same frequency band is shared by wake-up transceivers and WLAN modules, which brings about the co-existence problem. As for the $2.4 \mathrm{GHz}$ wake-up receiver suggested for sensor networks [8], because sensor nodes usually are deployed in remote environments and data signals are sent infrequently, the mutual co-channel interference between data signals and wake-up signals is seldom a big concern and is not touched. In contrast, WLAN signals are far overwhelming compared with wake-up signals and their coexistence is a big issue. In this design, wake-up signals are transmitted on the same channel as WLAN signals, using a compatible MAC protocol. This co-existence mechanism is different from the one studied in [20], where orthogonal channels are used.

\subsection{Tradeoff between simplicity (low power) and reliability}

A wake-up receiver is kept awake for a long period during which the host AP is in sleep. Therefore, we aim at realizing reliable wake-up signaling between nodes and APs via simple wake-up transceivers, with the following design targets.

The first target, of course, is low power consumption. To this end, a wake-up receiver is made as simple as possible so as to run at an extremely low power. A traditional receiver, adopting the super-heterodyne architecture, consumes much power for the RF oscillator in frequency conversion. A tuned RF with direct RF envelope detection eliminates the need for a local oscillator [7]. In this article, a similar architecture is adopted. (i) OOK and RF envelope detection are used instead of coherent detection; (ii) Error correction is not implemented, and ID matching is based on correlation.

The second target is reliability. Simplicity might degrade system reliability. (i) Co-channel interference. Wake-up signals, under OOK modulation, have OFF periods, which may be regarded as a clear channel by nearby WLAN devices, and interrupted by new transmission of a WLAN signal. (ii) Adjacent channel interference. It is usually difficult to produce narrow-band $\mathrm{BPF}$ in the RF band. As a result, the RF BPF is not very sharp at the band edge, and a wake-up signal extracted with such a BPF is susceptible to interference from WLAN signals on adjacent channels. The interference is especially obvious when the wake-up signal is recovered by non-coherent envelope detection. (iii) False wake-up probability. Without error correction, wake-up events may be falsely triggered by WLAN signals. These problems are specific to ROD WLANs.

\subsection{Budget of power consumption of wake-up receiver}

In this section we provide a simple analysis on power consumption of the AP system. Let duty ratio of an AP be $O_{\text {AP }}$, during which the main system of the AP is turned on and its wake-up receiver is turned off. The main system of the AP consumes a power of $P_{\mathrm{AP}}$, which includes power consumption of CPU, memory, etc. besides the power taken by the WLAN card for transmitting packets. In the rest $1-O_{\mathrm{AP}}$ period, the main system of the AP is put into sleep and the wake-up receiver is turned on, with power consumption of $P_{\mathrm{WuRcv}}$. Occasionally, because of the interference, the wake-up receiver may falsely activate the main system of the AP with a 
small probability FPP. Power consumption ratio of the wake-up receiver, $r_{\mathrm{WuRcv}}$, is computed as follows

$$
r_{\mathrm{WuRcv}}=\frac{P_{\mathrm{WuRcv}} \cdot\left(1-\mathrm{O}_{\mathrm{AP}}\right)}{P_{\mathrm{AP}} \cdot O_{\mathrm{AP}}+P_{\mathrm{WuRcv}} \cdot\left(1-\mathrm{O}_{\mathrm{AP}}\right)+P_{\mathrm{AP}} \cdot \mathrm{FPP} \cdot\left(1-\mathrm{O}_{\mathrm{AP}}\right)},
$$

where the numerator is the average power consumed by the wake-up receiver and the denominator is the average power consumed by the whole AP system. With a typical power consumption level $P_{\mathrm{AP}}=5 \mathrm{~W}$ and FPP $=$ $10^{-10}$, curves of $r_{\mathrm{WuRcv}}$, corresponding to three typical values of $P_{\mathrm{WuRcv}}$, are shown in Figure 1. When a Zigbee device is used as the wake-up receiver [3,12], it consumes tens of milliwatts $(>10 \mathrm{~mW})$. A wake-up receiver designed for sensor nodes should consume less than $0.1 \mathrm{~mW}$ [21]. According to Figure 1, $r_{\mathrm{WuRcv}}$ increases with $P_{\mathrm{WuRcv}}$. $r_{\text {WuRcv }}$ at $10 \mathrm{~mW}$ is relatively high and it is higher for a Zigbee device consuming more than $10 \mathrm{~mW}$. In addition, the transmission range of Zigbee devices is usually less than $30 \mathrm{~m}$ [12], which cannot meet the communication requirement of WLANs. Therefore, it is not suitable to use a Zigbee device as the wake-up receiver for ROD WLANs. Although $r_{\mathrm{WuRcv}}$ at $0.1 \mathrm{~mW}$ is low, it requires complex design in hardware. In comparison, $1 \mathrm{~mW}$ is a realistic design target. When duty ratio of an AP equals 0.04 (the AP is used for about $1 \mathrm{~h}$ per day), the wake-up receiver, at the power level of $1 \mathrm{~mW}$, consumes no more than $1 \%$ of the total power, which is satisfactory. Furthermore, such a wake-up receiver can be easily implemented with present technologies.

The ratio of the power consumption of a ROD AP system (power consumption is the denominator of Equation (1) by using a wake-up receiver) to that of a conventional AP (without a wake-up receiver and always powered on) is $\eta=O_{\mathrm{AP}}+P_{\mathrm{wuRcv}} / P_{\mathrm{AP}} \cdot\left(1-O_{\mathrm{AP}}\right)+\mathrm{FPP} \cdot\left(1-O_{\mathrm{AP}}\right) \cdot \eta$ decides how much the wake-up receiver can reduce the

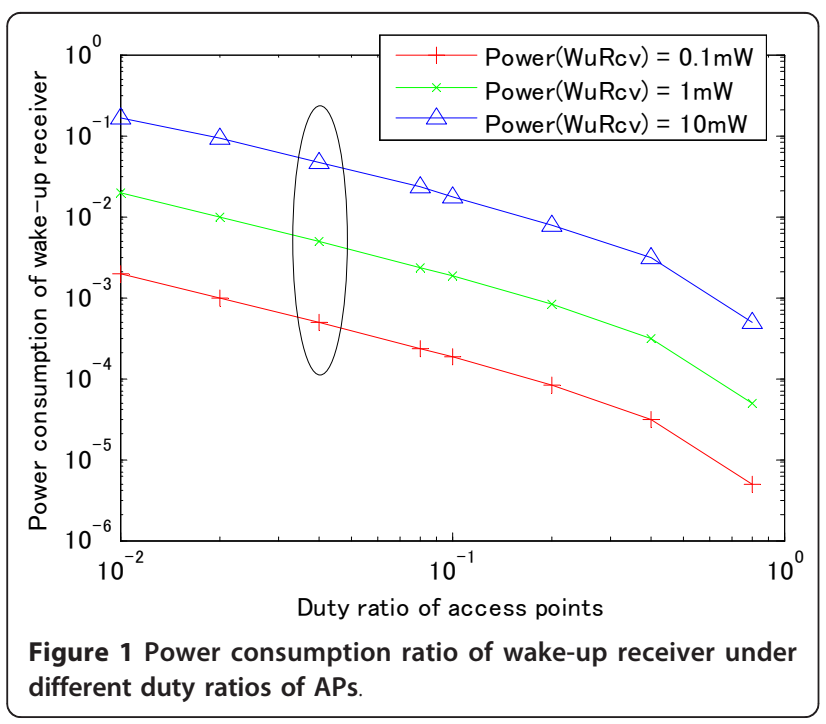

power consumption. Its third term is usually negligibly small and its second item is also very small. As a result, $\eta$ mainly depends on $O_{\text {AP }}$ and equals to 0.0402 when $O_{\mathrm{AP}}=0.04, P_{\mathrm{WuRcv}}=1 \mathrm{~mW}$, and $P_{\mathrm{AP}}=5 \mathrm{~W}$.

\subsection{Brief summary}

\subsubsection{Design freedom}

Compared with sensor networks where nodes run with battery and have a strict power budget, APs in ROD WLANs have stable power supply and the power consumption of a wake-up receiver can be relaxed a little. In ROD WLANs, a wake-up transmitter works occasionally whose power consumption is much less than that of the main system. Then, part of the complexity of a wake-up receiver can be shifted to the wake-up transmitter.

\subsubsection{Design challenge}

Different from sensor networks where data signals are sent infrequently and interferences seldom occur, WLAN signals are overwhelming in ROD WLANs and cause many interferences, especially when an imperfect RF BPF is used to extract wake-up signals. Therefore, a wake-up transceiver of ROD WLAN should deliver wake-up signals reliably and have a very low false wakeup probability.

In our previous work [18], we have already studied the signal co-existence problem and also studied how to detect wake-up signals and how to distinguish wake-up signals from WLAN signals with HardDec. Signal coexistence is realized at the wake-up transmitter, while detecting and distinguishing wake-up signals are realized at the wake-up receiver. In this article, the same wakeup transmitter is used. However, at the receiver, we consider the effect of an imperfect RF BPF and suggest SoftDec for detecting wake-up signals and distinguishing wake-up signals from WLAN signals. For the completeness, the system model of wake-up transceiver and the transmission procedure are briefly described.

\section{Wake-up transceiver for ROD WLANs}

In this section, we present the detailed design of the wake-up transceiver. First, the system model of the ROD WLANs is defined in Section 3.1. Then, transmission and reception of wake-up signal are described in Sections 3.2 and 3.3, respectively. Next, the necessity of and the method to distinguishing wake-up signals from WLAN signals are explained in Section 3.4 and the performance analysis is given in Section 3.5. In the detection and recognition of wake-up signals, adjacent channel interference is taken into account.

\subsection{System model}

In the proposed system model for ROD WLANs, a node (STA) is connected to the network via its associated AP. 
Besides WLAN cards, both the STA and the AP are equipped with an additional wake-up transceiver. The WLAN modules perform normal data communications between the AP and its associated STA, while the wakeup transceiver conveys wake-up signals from the STAs to their AP. Figure 2 shows the diagram of a wake-up transceiver, which is composed of two parts, with the top being the transmitter (WuTx) and the bottom being the receiver (WuRcv).

An AP in the idle state, before entering the sleep mode, notifies the sleep event to its associated STAs, and sets up its WuRcv to monitor a predetermined channel. Then, main system of the AP and WuTx are put into sleep, while the LAN module and WuRcv are kept awake, to accept wake-up requests from either the network side (wake-on-LAN) [16] or the STA (via WuRcv). The latter is the focus of our research.

A wake-up signal carries a WID, which is computed from the ID of an AP. Each AP has an ESSID (a variable length of ASCII characters), which is a group ID shared by all APs in the same ESS (extended service set). Each AP also has a unique BSSID (the 6bytes MAC address of a WLAN card) to identify its BSS (basic service set). Computation of the WID, via a well-known hash function $h$ (e.g., SHA-1 used for 802.11i [22]), is done as follows:

WID $=$ A predetermined WID, ESSID not known,

WID $=h\left(\mathrm{AP}^{\prime}\right.$ ' ESSID $)$, ESSID known but not associated,

$\mathrm{WID}=h\left(\mathrm{AP}^{\prime}\right.$ s ESSID || $\mathrm{AP}$ 's BSSID $)$, ESSID known \& associated,

where $\|$ is the concatenation operation. An exception is that when ESSID of the target AP is unknown, a predetermined WID will be sent to activate all APs and this is actually a broadcast WID. WuTx of a STA transmits a proper WID according to its present state. WuRcv of an AP should store all three WIDs so as not to miss any wake-up requests.

\subsection{Transmission of wake-up signals}

The wake-up procedure starts when a STA wants to access the external network via an AP, which happens to be in the sleep mode.

First, WuTx of the STA computes a WID of the target AP according to Equation (2). The WID is prepended by a sequence of $N$ preamble bits, and then Manchester coded (MC) using the same MC codes (bit 0 is encoded to $(1,0)$ and bit 1 is encoded to $(0,1))$ as in IEEE 802.3 [23]. The carrier frequency is spread with chirp spread spectrum (CSS) [24] so as to satisfy the spectral density and bandwidth requirements of FCC [25] and MIC [26]. Then, the carrier signal is pulse modulated by the MC coded bits. WuTx of the STA transmits the generated wake-up signal when the channel is sensed as being idle. In this process, the WID is carried in the envelope, which is not affected by the CSS operation. Figure 3 shows an example of the generated wake-up signal.

To enable co-existence of wake-up signals with WLAN signals (avoid co-channel interference), a wakeup signal is transmitted by a protocol compatible with CSMA/CA, as shown in Figure 4. (i) WuTx performs carrier sense before transmission. (ii) WuTx prevents WLAN signals from breaking in (retain the channel) by controlling the length of OFF periods. As shown in Figure 3, there are continuous OFF periods in an OOK

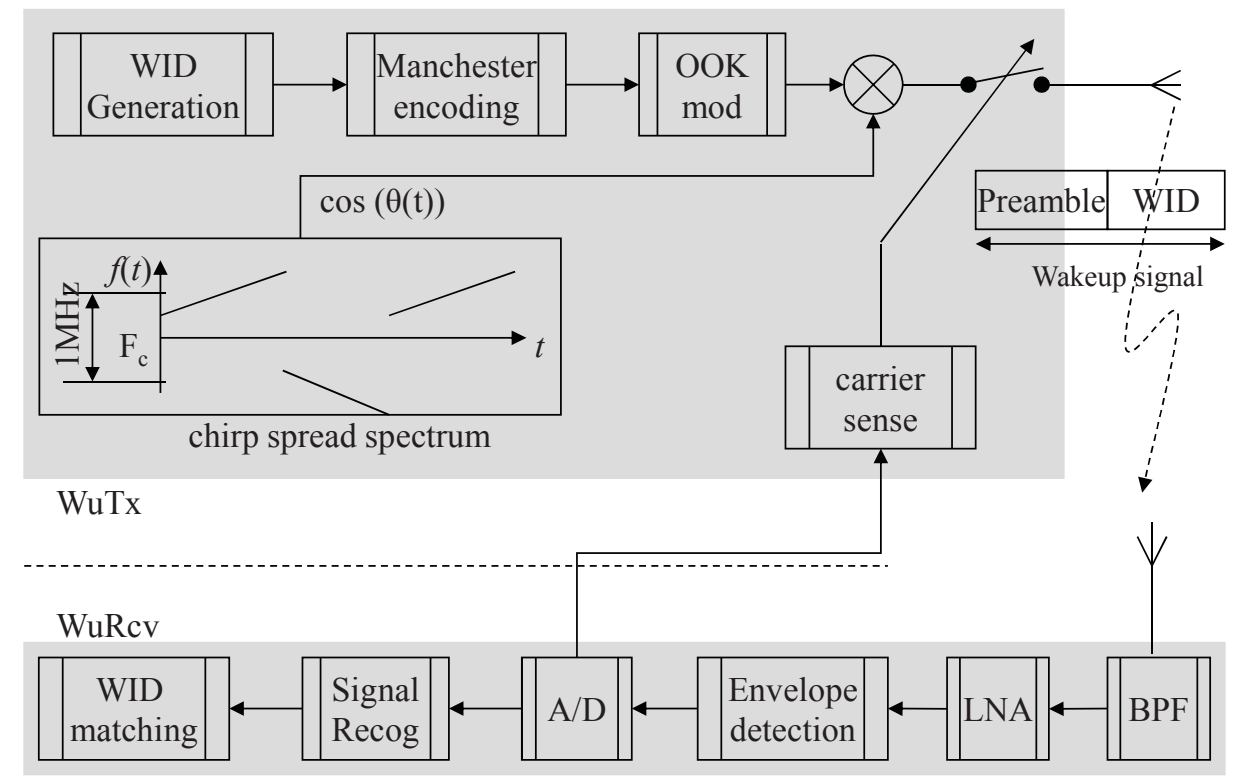

Figure 2 Diagram of wake-up transceivers. 


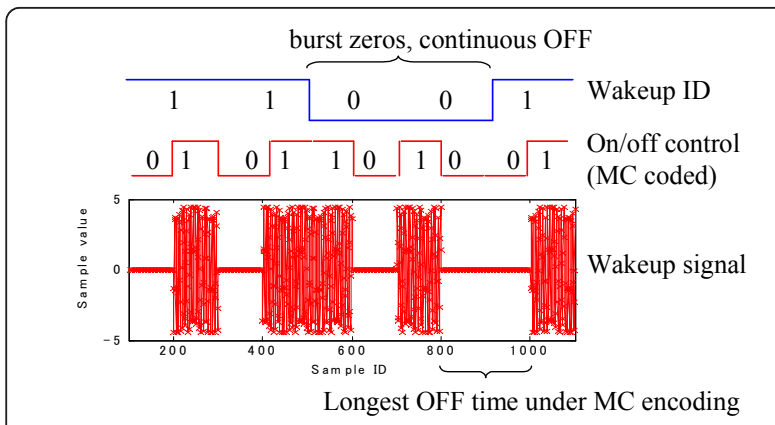

Figure 3 An example of wake-up signals

modulated wake-up signal. With the MC codes, the continuous OFF time is limited to no more than one bit long. To further ensure that the transmission of a wakeup signal will not be corrupted by WLAN signals, the transmit rate of a wake-up signal is restricted so that even the longest OFF time under MC encoding will not exceed the minimal arbitration inter-frame space (AIFS) [22]. With minimal AIFS $=19$ us in $802.11 \mathrm{~g}$, the bit rate should be greater than $50 \mathrm{kbps}$. Experimental results indicate that $100 \mathrm{kbps}$ is a good choice.

\subsection{Reception of wake-up signals}

WuRcv of the AP extracts the wake-up signal with a RF $\mathrm{BPF}$, followed by amplification, envelope detection and WID matching. WID matching is performed only when a signal is recognized as a wake-up signal. If the detected WID matches the assigned one, a wake-up event is triggered to activate the main system of the AP.

\subsubsection{BPF and LPF}

The bandwidth of a wake-up signal, containing 95\% energy, is about $1 \mathrm{MHz}$. Although the BPF bandwidth at a WuRcv should be set to this value, in this design, it is set to that of a WLAN channel $(20 \mathrm{MHz})$ for the following reasons: (i) total energy of a WLAN channel is monitored for the purpose of carrier sense. (ii) The envelope of WLAN signals is used in signal recognition. A BPF with a narrower bandwidth changes the distribution of WLAN signals envelope and increases the false probability of signal recognition, as is confirmed by initial experiments.

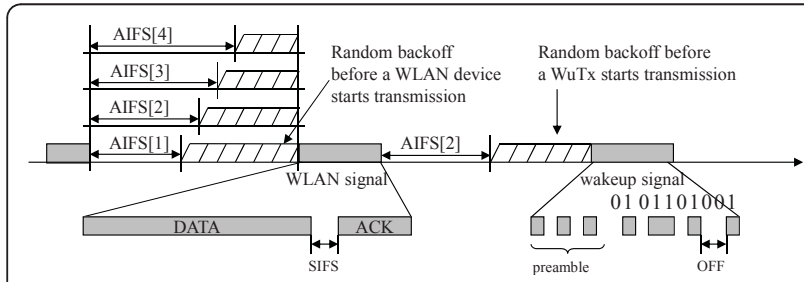

Figure 4 Channel sharing between WLAN signals and wake-up signals.
Since the bandwidth of BPF is much greater than that of wake-up signals, much noise survives the BPF filter and is involved in the envelope. The subsequent low pass filter (LPF, bandwidth is set to $5 \times$ symbol rate), used for smoothing envelopes, can remove some of the out-of-band thermal noise. By simulation, we confirmed that with the following conditions, bit rate $=100 \mathrm{kbps}$, LPF bandwidth $=1 \mathrm{MHz}, \mathrm{BPF}$ bandwidth $=20 \mathrm{MHz}, \mathrm{LPF}$ in the non-coherent envelope detection has a SNR gain of $8 \mathrm{~dB}$, compared with $13 \mathrm{~dB}$ in the coherent detection. In other words, non-coherent detection causes $5 \mathrm{~dB}$ SNR loss compared with coherent detection. In addition, this SNR loss increases with the rate since a larger LPF bandwidth is required at a higher rate.

\subsubsection{Effect of an imperfect BPF}

In the previous discussion, we did not consider adjacent channel interference, with an implicit assumption that the BPF can completely remove out-of-band interference. In WLANs, a RF BPF usually has a bandwidth as large as the whole ISM band (e.g., $83.5 \mathrm{MHz}$ for 2.4 GHz ISM band). The filtered RF signal is down-converted to the baseband, where a LPF is used to extract signals from a specific channel. The LPF can be made very sharp. However, as shown in Figure 5, a practical BPF with $20 \mathrm{MHz}$ RF bandwidth is imperfect: its slope is not sharp. As a result, out-of-band interferences may be involved in the envelope of wake-up signals.

Wireless local area network signals can be transmitted in parallel on orthogonal channels (separated by $25 \mathrm{MHz}$ or more) without mutual interference. But with an imperfect BPF, WLAN signals on a channel $\left(F_{c}+\right.$ $25 \mathrm{MHz}$, e.g., $\mathrm{CH} 6)$ may leak to a neighboring channel $\left(F_{c}\right.$, e.g., $\left.\mathrm{CH} 1\right)$ where a wake-up receiver is monitoring the channel. An imperfect BPF has two effects. (1) The

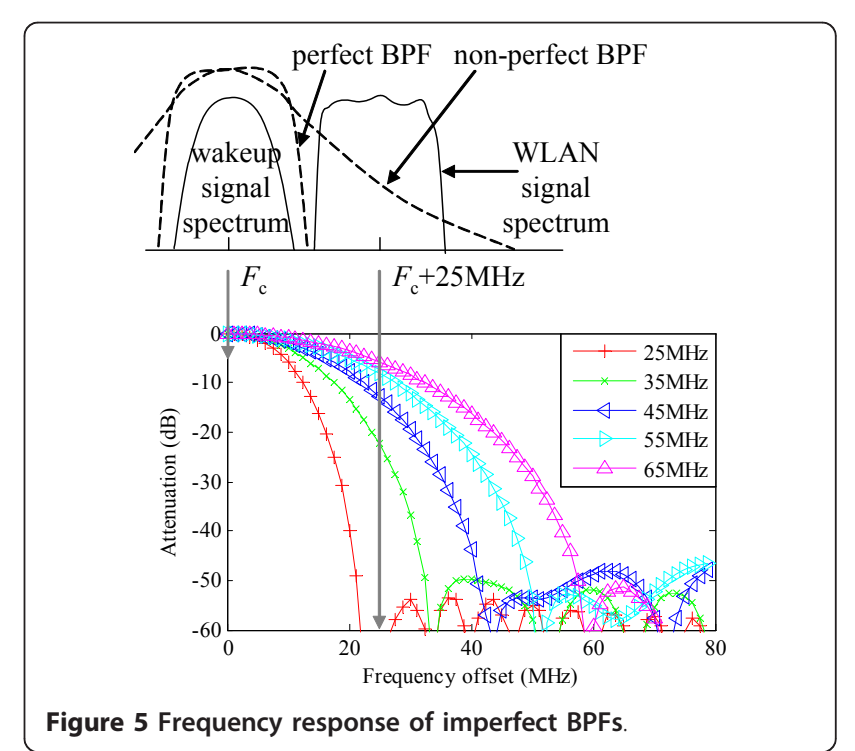


number of false wake-up events may be increased since WLANs signals on adjacent channels are also received by the wake-up receiver. (2) Frame error rate may be increased. The OFF period of an OOK modulated wakeup signal means 0 . But in the presence of interference, leaked energy will increase the signal energy in the OFF period, which leads to higher bit error rate.

\subsubsection{WID matching}

The envelope after LPF is normalized by the automatic gain control (AGC) operation, and sampled to MC codewords, each of which contains two adjacent envelope samples. The $n$th $\mathrm{MC}$ codeword is $\left(r_{n, 1}, r_{n, 2}\right)$ (refer to Figure 6a). MC codes are simple DSSS codes with the length equaling to 2 . Therefore, SoftDec is possible. The $n$th MC codeword is quantized to $\left(\hat{r}_{n, 1}, \hat{r}_{n, 2}\right)$ using multiple bits, and the SoftDec follows Equation (3).

$$
\begin{aligned}
& \operatorname{corr}_{n, 0}=\hat{r}_{n, 1} \cdot(1)+\hat{r}_{n, 2} \cdot(-1)=-\left(\hat{r}_{n, 2}-\hat{r}_{n, 1}\right), \\
& \operatorname{corr}_{n, 1}=\hat{r}_{n, 1} \cdot(-1)+\hat{r}_{n, 2} \cdot(1)=\left(\hat{r}_{n, 2}-\hat{r}_{n, 1}\right), \\
& \operatorname{corr}_{n, 1} \leq-\Delta \rightarrow \operatorname{WID}_{n}=0 ; \operatorname{corr}_{n, 1} \geq \Delta \rightarrow \operatorname{WID}_{n}=1,
\end{aligned}
$$

otherwise, bit error.

The role of $\Delta$ in SoftDec will be discussed later. With a bit decision threshold $\beta$, the corresponding bit $\mathrm{WID}_{n}$ can also be hard decoded to 0 or 1 according to Equation (4).

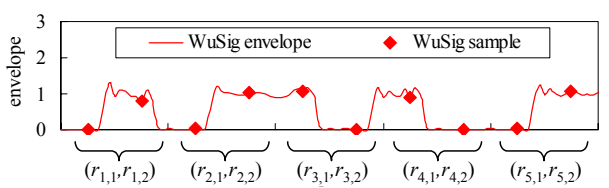

(a) envelope samples of wakeup signals

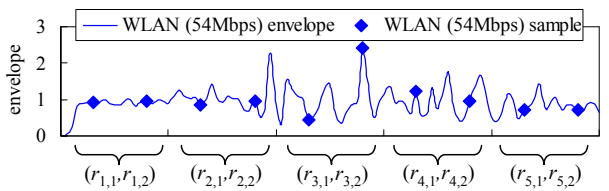

(b) envelope samples of WLAN signals

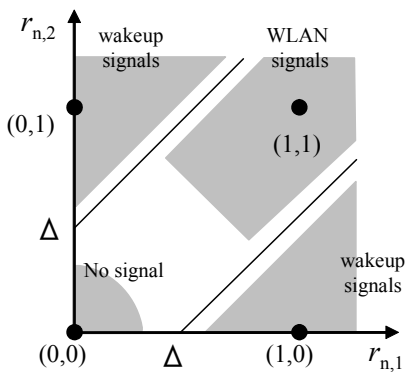

(c) signal space of $\left(r_{\mathrm{n}, 1}, r_{\mathrm{n}, 2}\right)$

Figure 6 Envelope of wake-up signals and WLAN signals; Signal space and signal recognition

$$
\begin{aligned}
& r_{n, 1}>\beta, \quad r_{n, 2}<\beta \rightarrow \mathrm{WID}_{n}=0, \\
& r_{n, 1}<\beta, \quad r_{n, 2}>\beta \rightarrow \mathrm{WID}_{n}=1,
\end{aligned}
$$

otherwise, bit error.

The received WID is compared with the assigned WID and a wake-up event is triggered if the WID matches. In the subsequent evaluations, we will further show that HardDec is sufficient when there is no interference. But it is difficult to find a fixed $\beta$ applicable to all scenarios after taking the potential adjacent channel interference into account. In contrast, SoftDec helps to resist adjacent channel interference leaked by an imperfect BPF.

\subsection{Signal recognition}

For the simplicity, error correction is not implemented. As a result, the envelope of a WLAN signal may falsely match the assigned WID, when actually there is no communication demand. This is solved by signal recognition, which is necessary because there are many more WLAN signals than wake-up signals on the shared channel. It is based on the clear distinction in envelope distribution of wake-up signals and WLAN signals. Figure 6 shows an example of envelopes of a wake-up signal and a WLAN signal (54 Mbps, orthogonal frequency division multiplexing (OFDM), 64QAM, coding rate = $3 / 4$ ), respectively. Figure 6 a corresponds to the wake-up signal in Figure 3. One sample of a MC codeword $\left(r_{n, 1}\right.$, $\left.r_{n, 2}\right)$ is around 0 and the other is around 1 , with a clear difference in envelope values. In comparison, most samples in Figure $6 \mathrm{~b}$ are around 1 although some samples may deviate greatly due to the well known peak-to-average power ratio (PAPR) problem of OFDM modulation [27]. Figure 6c shows the signal space of $\left(r_{n, 1}, r_{n, 2}\right)$. Points $(1,0)$ and $(0,1)$ correspond to wake-up signals, point $(1,1)$ corresponds to WLAN signals, and point $(0$, 0 ) corresponds to silent channel without any transmission. Division of the signal space according to SoftDec in Equation (3) is also shown in Figure 6c.

$$
y_{n}=\left|\hat{r}_{n, 1}-\hat{r}_{n, 2}\right| \geq \Delta
$$

as a logic variable, is used to indicate whether a codeword is a valid MC codeword or not, where each envelope sample is represented by multiple bits after quantization in times of SoftDec. In the ideal case, $y_{n}$ is 1 for a wake-up signal and 0 for a WLAN signal. To improve the reliability, $y_{n}$ is computed from each of the first $N$ codewords (the preamble part of wake-up signals, or the leading part of WLAN signals), and its average is used as the discriminative metric-Manchester code rate (MCR),

$$
\mathrm{MCR}=\frac{1}{N} \sum_{n=1}^{N} y_{n}
$$


For a received signal, its MCR is computed and compared against a fixed threshold $\alpha$. The signal is regarded as a wake-up signal if MCR $>\alpha$, and a WLAN signal otherwise, as follows:

$$
\text { MCR }>\alpha \rightarrow \text { WuSig, MCR }<\alpha \rightarrow \text { WLAN Sig. }
$$

\subsection{False probability analysis}

In this section, we analyze the false probability of signal recognition. Using Equation (7) in signal recognition, it is equivalent to compare $\sum_{n=1}^{N} y_{n}$ against $N \cdot \alpha$. A false positive event occurs when a WLAN signal is recognized as a wake-up signal, i.e., $\sum_{n=1}^{N} y_{n}>N \cdot \alpha$ for a WLAN signal. A false negative event occurs when a wake-up signal is recognized as a WLAN signal, i.e., $\sum_{n=1}^{N} y_{n}<N \cdot \alpha$ for a wake-up signal. In the analysis, we assume that a WLAN signal is longer than the preamble of a wake-up signal, which is true for typical data transmissions.

With the assumption that each envelope sample is independent of others, $y_{n}, n=1,2, \ldots, N$, is a Bernoulli process [28]. The error probabilities, $\operatorname{prob}\left(y_{\mathrm{n}}=1\right.$ | WLAN) for a WLAN signal and $\operatorname{prob}\left(y_{n}=0 \mid \mathrm{WuSig}\right)$ for a wake-up signal, can be obtained from experimental data. Then, the false positive probability (FPP) for a WLAN signal and false negative probability (FNP) for a wake-up signal can be computed according to Equations (8) and (9), respectively.

$$
\begin{aligned}
\operatorname{FPP}_{\text {WLAN }}(N, \alpha) & =\sum_{k=|N \alpha|+1}^{N} C_{N}^{k} \cdot p^{k} \cdot(1-p)^{N-k}, \\
p & =\operatorname{prob}\left(y_{n}=1 \mid \text { WLAN }\right) . \\
\operatorname{FNP}_{\text {WuSig }}(N, \alpha) & =\sum_{k=N-|N \alpha|+1}^{N} C_{N}^{k} \cdot p^{k} \cdot(1-p)^{N-k}, \\
p & =\operatorname{prob}\left(y_{n}=0 \mid \mathrm{WuSig}\right) .
\end{aligned}
$$

With $\operatorname{prob}\left(y_{n}=0 \mid \mathrm{WuSig}\right)=10^{-4}, \operatorname{prob}\left(y_{n}=1 \mid \mathrm{WLAN}\right)$ $=10^{-1}$, and $\alpha=0.8$, FPP and FNP are computed under different $N$ and shown in Figure 7. This figure shows that FPP decreases exponentially fast as $N$ increases and FNP does in a similar way. Then, a sufficiently small FPP/FNP can be achieved by choosing a suitable $N$, e.g., $N=20$. Since $N$ is the number of preamble bits, this property ensures that signal recognition can be realized by low overhead when a small $N$ is sufficient. In this system, signal detection depends on $\Delta$, and signal recognition depends on $N, \alpha$ and $\Delta$. With $N$ dominating FPP/

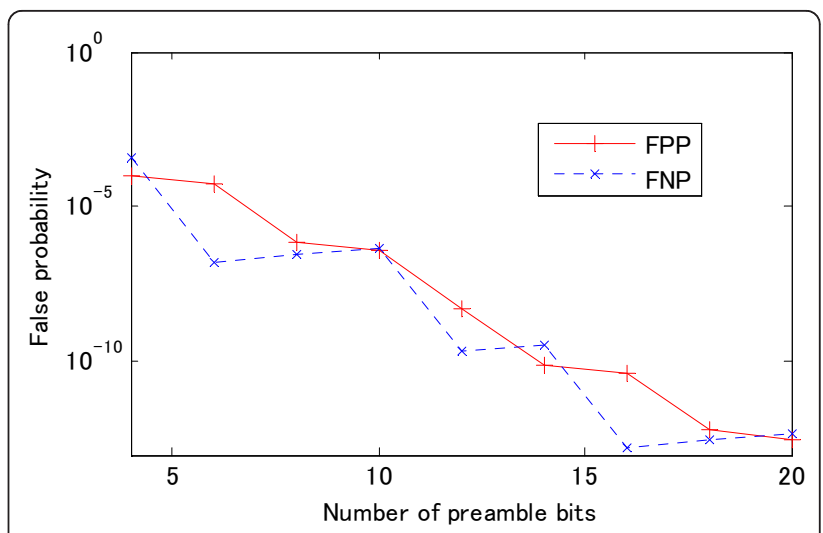

Figure 7 FPP/FNP under different numbers of preamble bits.

FNP, $\Delta$ can be adjusted to meet the performance of signal detection. This is another point of Figure 7.

\section{Evaluation and analysis}

In this section, we first evaluate the effect of an imperfect BPF and the performance of the proposed scheme under different BPF bandwidths by simulation. Then, we also evaluate the proposed scheme with a simple testbed. These days most WLAN devices in $2.4 \mathrm{GHz}$ use $802.11 \mathrm{~g}$. It is required in [22] that the lowest rate (6 Mbps) of OFDM modulation in $802.11 \mathrm{~g}$ should be supported at RSSI $=-82 \mathrm{dBm}$. Therefore, we will check whether the wake-up receiver can satisfy the same requirement. When evaluating false probability of signal recognition, 802.11g ERP-OFDM signals of eight rates are generated and processed by the same wake-up receiver.

We use the parameters in Table 2 unless stated otherwise. We further use the following settings when evaluating the effect of imperfect BPFs.

- Channel. WLAN signals can be transmitted in parallel on two orthogonal channels (e.g., $\mathrm{F}_{\mathrm{c}}=\mathrm{CH} 1$ and $\mathrm{F}_{\mathrm{c}}+25 \mathrm{MHz}=\mathrm{CH} 6$ in Figure 5) without mutual

Table 2 Default parameters used in experiments

\begin{tabular}{ll}
\hline Parameter & Default value \\
\hline Bit rate of wake-up signal & $100 \mathrm{kbps}$ \\
Length of preamble & 20 bits \\
Length of wake-up ID & $128 \mathrm{bits}$ \\
WLAN signals & $802.11 \mathrm{~g}$ ERP-OFDM, 8rates \\
Distance of adjacent channels & $25 \mathrm{MHz}$ \\
Signal to interference ratio & $0 \mathrm{~dB}$ \\
Noise power & $-91 \mathrm{dBm}$ per 20MHz \\
Bandwidth of RF band pass filter & $65 \mathrm{MHz}$ at 10dB attenuation \\
Number of runs per evaluation & 10,000 \\
\hline
\end{tabular}


interference. Hence, we assume that a wake-up signal is transmitted on the frequency $F_{c}$ and the interfering WLAN signal is transmitted at 54 Mbps on the frequency $\mathrm{F}_{\mathrm{c}}+25 \mathrm{MHz}$.

- Signal power. By default, we consider the case where an interfering WLAN signal arrives at the WuRcv with the same RSSI as the wake-up signal. We will also evaluate the effect of different signal to interference ratio (SIR).

- BPF. At the WuRcv, the interfering WLAN signal on $\mathrm{F}_{\mathrm{c}}+25 \mathrm{MHz}$ is not sufficiently attenuated by the $\mathrm{BPF}$, and the amount of remaining interference depends on the BPF bandwidth. Several BPF filters, whose $10 \mathrm{~dB}$ bandwidths are $25,35,45,55$, and 65 $\mathrm{MHz}$, are generated to mimic the different degrees of interference due to incomplete attenuation, and their frequency responses are shown in Figure 5. With SIR equaling to OdB before BPF, the SIR after $\mathrm{BPF}$ is $34.6,16.5,10.7,7.4$, and $5.4 \mathrm{~dB}$, respectively. The last filter, with the bandwidth being $65 \mathrm{MHz}$, approaches off-the-shelf BPFs.

- Noise. In the evaluation, additive white Gaussian noise is assumed and its power is fixed at $-91 \mathrm{dBm} /$ $20 \mathrm{MHz}$, which is often used in network simulators such as QualNet [29].

\subsection{Envelope distribution and ideal SoftDec}

We first investigate the distribution of envelopes of WLAN signals and wake-up signals. Average envelopes with 95\% confidence interval, corresponding to WLANs signals transmitted at different rates, and ON parts and OFF parts of wake-up signals, are shown in Figure 8. This figure reveals two points. (1) WLAN signals have a larger deviation in envelope than wake-up signals. A further investigation shows that the deviation of WLAN

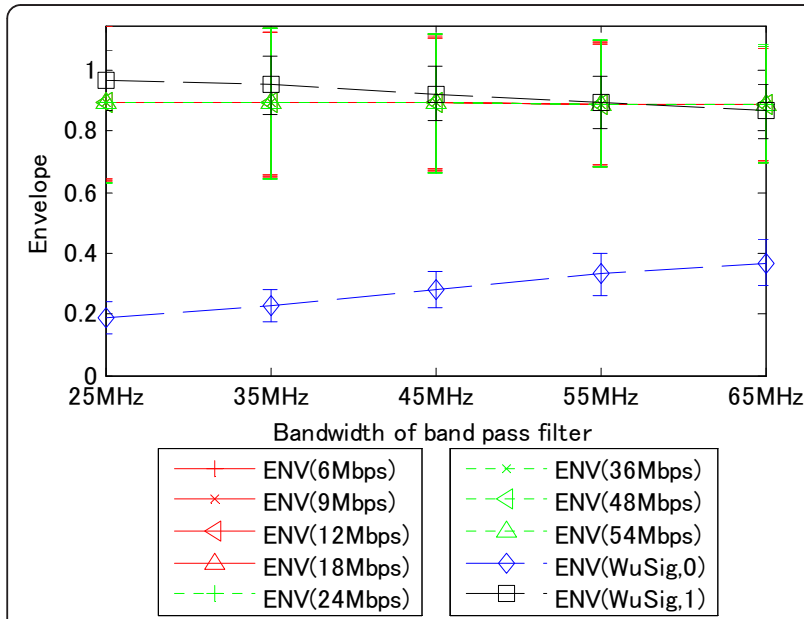

Figure 8 Average envelope samples under an imperfect BPF ( $95 \%$ confidence interval, RSSI $=-82 \mathrm{dBm}$ ). signal envelopes almost remains irrelevant of RSSI. This can be explained as follows: For WLAN signals with OFDM modulation, each sample is actually an addition of signals from many orthogonal sub-carriers (48 data sub-carriers and 4 pilot sub-carriers in $802.11 \mathrm{~g}$ OFDM). As a result, OFDM modulated WLAN signals have the well-known PAPR problem [27], where the energy (envelope) changes per-sample (refer to Figure 6b). (2) Average envelope of OFF parts of wake-up signals increases with BPF bandwidths while that of ON parts decreases. The former is because more interference energy is involved in the envelope with a larger BPF bandwidth. The latter is due to the operation of AGC. It is clear that a fixed threshold $\beta$ for the HardDec is not applicable to all scenarios. Next, we examine the effect of BPF bandwidth on frame error rate $\left(F^{2} R^{a}\right)$ of wake-up signals. Here we compare FER achieved by HardDec (HardDec, with decision threshold $\beta=0.5$ ) and ideal SoftDec under an imperfect BPF. An ideal condition is assumed for the SoftDec: no A/D bits limit and $\Delta=0$ is used for optimizing the performance of wake-up signal detection. The FER results are shown in Figure 9. According to this figure, performance of HardDec is more susceptible to the BPF bandwidth (interference increases with BPF bandwidth) compared with SoftDec, especially at a large bandwidth. As a result, SNR gain provided by SoftDec in comparison with HardDec increases with BPF bandwidths, ranging from about $3.5 \mathrm{~dB}\left(\mathrm{FER}=10^{-2}\right)$ at $25 \mathrm{MHz}$ to over $9 \mathrm{~dB}$ (FER $\left.=10^{-1}\right)$ at $65 \mathrm{MHz}$.

\subsection{Practical SoftDec}

In the above results, $\Delta=0$ is the best for SoftDec and offers the max SNR gain compared with HardDec. But

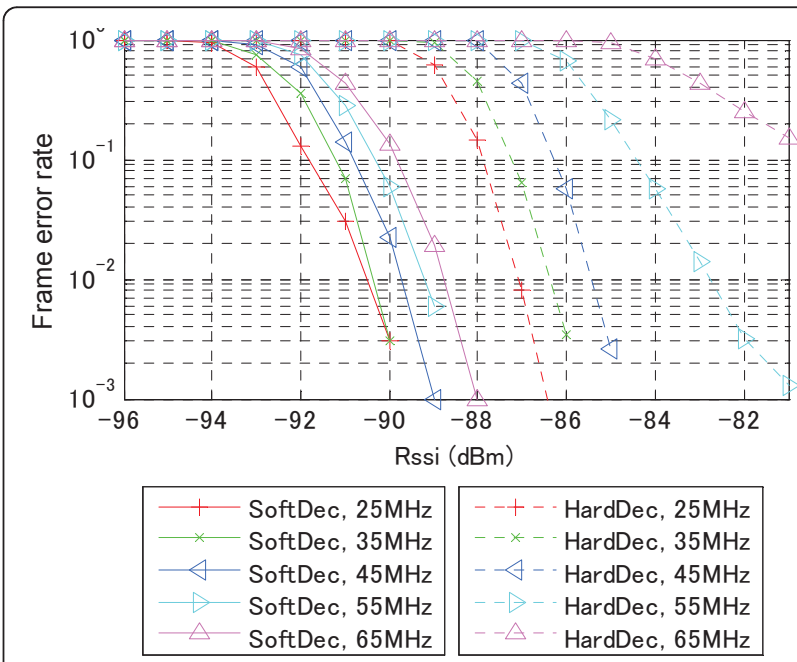

Figure 9 FER: SoftDec (no A/D bits limit, $\Delta=0$ ) vs. HardDec. 
in order to distinguish wake-up signals from WLAN signals, a non-zero $\Delta$ is necessary.

Selection of $\Delta$ depends on the envelope difference inside a MC codeword. To make correct SoftDec according to Equation (3), the absolute difference of each $\mathrm{MC}$ codeword of an interfered wake-up signal should be greater than $\Delta$, while that of a WLAN signal should be less than $\Delta$. An investigation of the absolute envelope difference (see Equation (5)) is shown in Figure 10, cumulative distribution function (CDF) for interfered wake-up signals and complementary CDF (CCDF) for WLAN signals, where the BPF bandwidth equals $65 \mathrm{MHz}$. At a given envelope difference threshold, the CDF of wake-up signals corresponds to $\operatorname{prob}\left(y_{n}=0 \mid \mathrm{WuSig}\right)$ and CCDF of WLAN signals corresponds to $\operatorname{prob}\left(y_{n}=1 \mid\right.$ WLAN), both representing MC code error rate under SoftDec. Without interference, the CDF/CCDF value of the cross point should be very low, where a $\Delta$ can ensure a low error rate for both wake-up signals and WLAN signals. But with interferences, the cross point has a relatively high error rate. It is clear that with a single $\Delta$, we cannot achieve low error rate for both detecting wake-up signals and distinguishing WLAN signals. FER of wake-up signals mainly depends on $\Delta$ while FPP/FNP also depends on other factors, $N$ and $\alpha$. According to Figure 7, FPP/ FNP decreases exponentially fast as $N$ increases. Therefore, in our design, $\Delta$ is chosen in an aggressive way to ensure a low error rate of wake-up signals. Then, $N$ and $\alpha$ are adjusted to satisfy FPP/FNP. Based on Figure 10, 0.25 is a good approximation of $\Delta$, where the envelope difference of an interfered wake-up signal is less than $\Delta$ with a low MC code error rate (on the order of $10^{-4}$ ).

The next question is how many bits should be used for the A/D conversion so as to accurately reflect the envelope

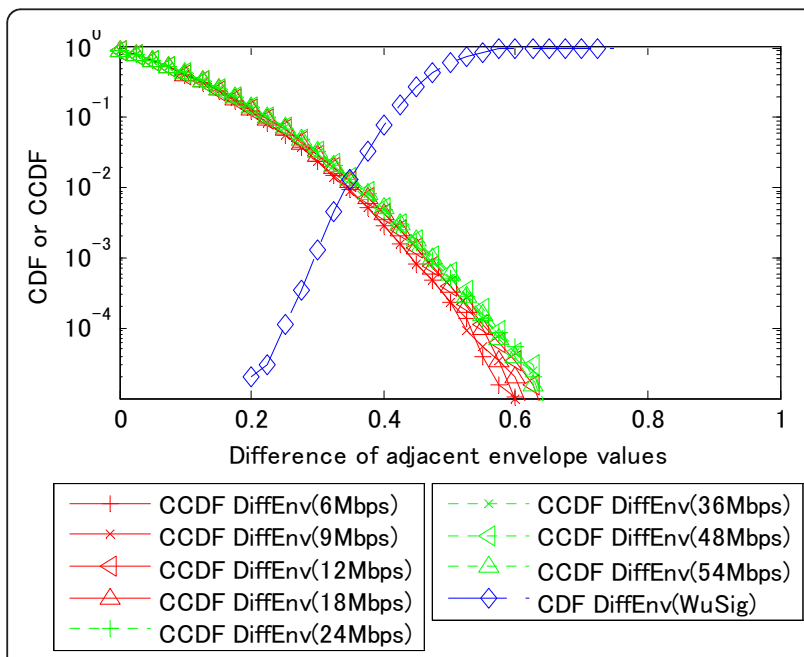

Figure 10 Distribution of difference of adjacent envelope samples (RSSI $=-82 \mathrm{dBm}, \mathrm{BPF}$ bandwidth $=65 \mathrm{MHz}$ ). difference shown in Figure 10. With AGC operation, the envelope is normalized to the range $0-1$. This range is equally divided into $2^{m}$ sub-ranges when using an $m$-bit A/D. By trying different numbers of sampling bits and different $\Delta$ values, we find that SoftDec with $m=2$ bits A/D and $\Delta=1 / 4$ achieves satisfactory FER results, as shown in Figure 11. Here $\Delta=1 / 4$ is consistent with the selection of the threshold based on Figure 10. A higher resolution A/D of course provides better results, but with higher cost. Compared with Figure 9, the SNR loss of SoftDec is about $6 \mathrm{~dB}\left(\mathrm{FER}=10^{-3}\right)$ when BPF bandwidth equals $65 \mathrm{MHz}$. This SNR loss is due to two factors: quantization and allowing a non-zero $\Delta$ for signal recognition.

\subsection{Signal recognition under SoftDec}

Signal recognition is conducted by comparing MCR of the $\mathrm{N}$-bit preamble of a received signal against a suitable threshold $\alpha . N=20$ is chosen according to Figure 7. To find a suitable threshold $\alpha$, the distribution of MCR is investigated. Figure 12 shows $\operatorname{CDF}(x)=\operatorname{prob}(\mathrm{MCR}<x)$ of wake-up signals and $\operatorname{CCDF}(x)=\operatorname{prob}(\mathrm{MCR}>x)$ of WLAN signals. At a given MCR threshold, CDF of wake-up signals is FNP and CCDF of WLAN signals is FPP. In the ideal case, MCR is 0 for a WLAN signal and 1 for a wake-up signal. Because $\Delta$ is chosen to ensure a low FER, MCR of wake-up signals is around 1. But MC error rate of WLAN signals is relatively high (on the order of $10^{-1}$ at $\Delta=1 / 4$ ). Then, MCR of WLAN signals is much greater than $0 . \alpha$ is set to 0.8 according to Figure 12. FPP and FNP are almost the same with $N=20$ and $\alpha=0.8$, as shown in Figure 7 .

Figure 13 shows the false probability of signal recognition achieved by SoftDec, which includes theoretical FPP of WLAN signals at eight different rates, theoretical FNP of wake-up signals, and FNP of wake-up signals obtained by simulation. FPP of simulation results is not shown here because we did not find any false positive events in the simulation. Theoretical results and simulation results of FNP match very well, which confirm the

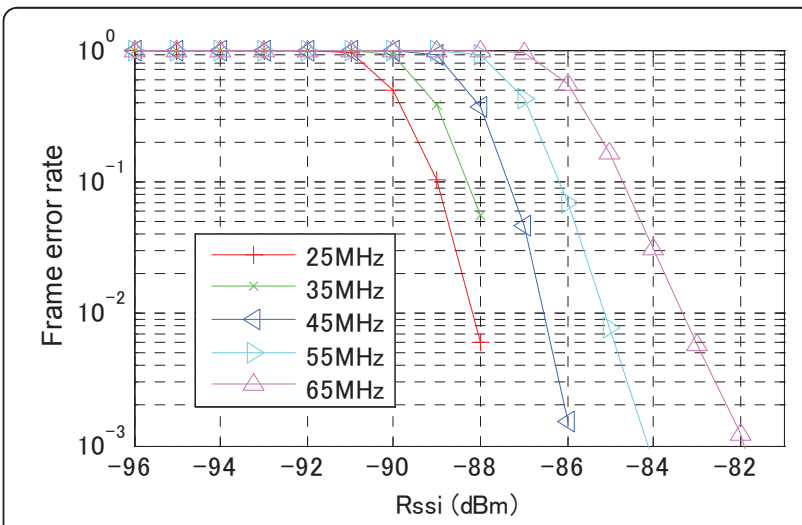

Figure 11 FER of SoftDec ( 2 bit $A / D, \Delta=1 / 4$ ) 


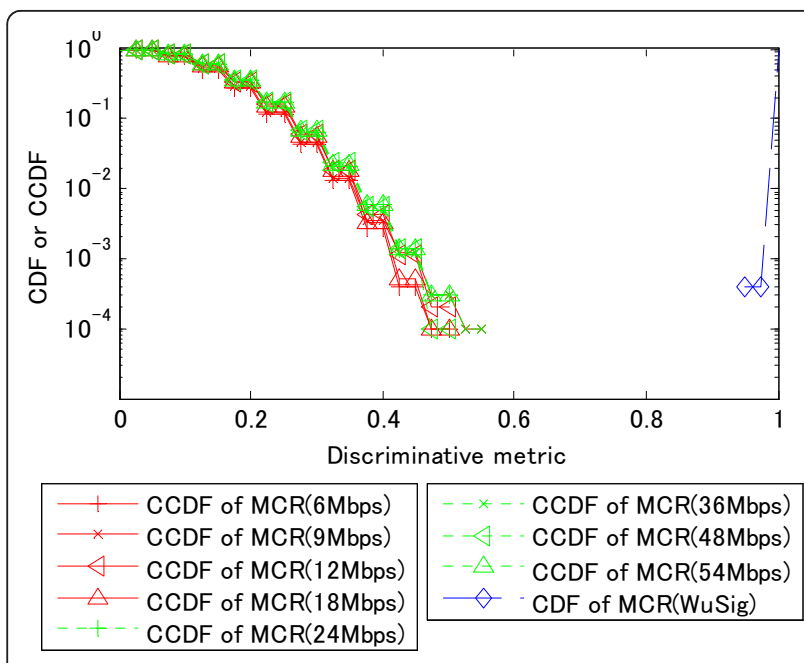

Figure 12 Distribution of MCR under SoftDec $(N=20,2$ bit A/ $\mathrm{D}, \Delta=1 / 4$, BPF bandwidth $=65 \mathrm{MHz}$ ).

accuracy of theoretical computation. Although FNP of wake-up signals decreases as RSSI increases, FPP increases with RSSI, which is contrary to intuitions. A further investigation shows that at low RSSI, noise involved in the envelope of WLAN signals can mitigate the PAPR problem in some degree. At higher RSSI, the PAPR problem gets serious, resulting in a little higher MC code error rate and FPP. However, as RSSI gets higher, FPP will approach a constant value because the effect of noise in envelope gradually disappears. Both FPP and FNP are satisfactory when RSSI $=-82 \mathrm{dBm}$.

\subsection{Effect of SIR}

In previous sections, SIR before BPF is fixed at OdB. In this section, the effect of SIR is examined. Figure 14

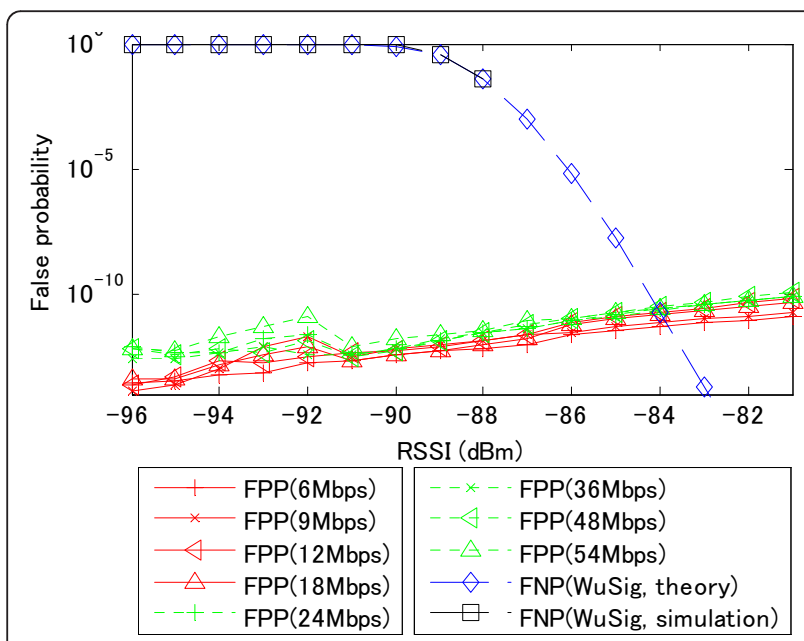

Figure 13 False probability under SoftDec $(N=20,2$ bit A/D, $\Delta$ $=1 / 4$, BPF bandwidth $=65 \mathrm{MHz}$ ).

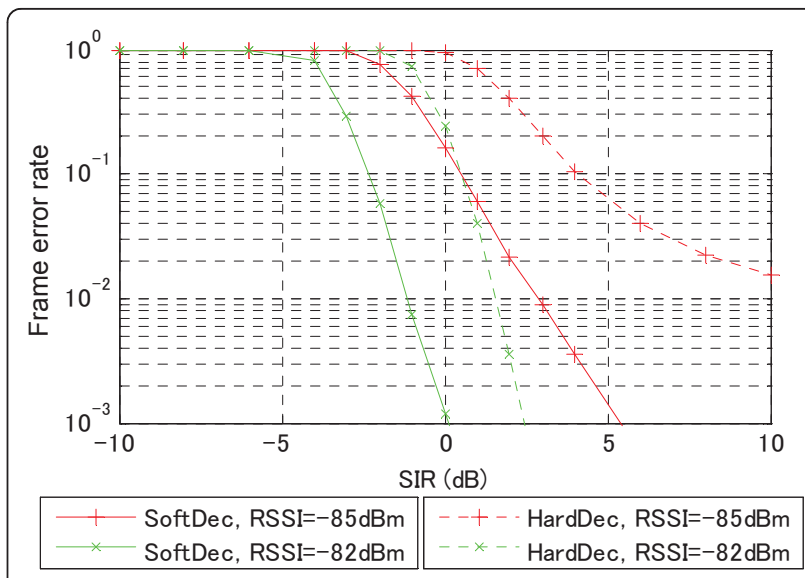

Figure 14 FER: SoftDec ( 2 bit $A / D, \Delta=1 / 4$ ) vs. HardDec under different SIRs (BPF bandwidth $=65 \mathrm{MHz}$ ).

shows FER of SoftDec and HardDec under different SIRs, where RSSI of wake-up signals is set to -82 or $-85 \mathrm{dBm}$. When RSSI of wake-up signals is $-82 \mathrm{dBm}$, SoftDec offers about $2.5 \mathrm{~dB}$ gain $\left(\mathrm{FER}=10^{-2}\right.$ ) compared with HardDec. It is interesting to see that when RSSI of wake-up signals decreases to $-85 \mathrm{dBm}$, the SNR gain of SoftDec against HardDec increases to over $7 \mathrm{~dB}$ (FER = $10^{-2}$ ). This is due to the following factor: At lower RSSI, thermal noise affects FER greatly; Thermal noise causes common interference to adjacent envelope samples, which SoftDec can effectively remove but HardDec cannot; As a result, HardDec has a lower slope rate in FER compared with SoftDec.

\subsection{FER of SoftDec on the testbed}

To measure the FER performance of SoftDec in the real system, we used the same testbed system as in [18], which is shown in Figure 15. Wake-up signals are transmitted by a signal generator (Anritsu MG3700A); RF and envelope detection of the wake-up receiver are based on USRP2 (XCVR2450 for WLAN) and GNU Radio [30]. Signal recognition and WID matching are processed by Matlab. Antennas of signal generator and USRP2 are connected via a co-axial cable to get the accurate RSSI setting. FER of SoftDec is evaluated with respect to different RSSI setting, where three transmit rates $(100,200,500 \mathrm{kbps})$ of wakeup signals are considered. As a comparison, we also show the FER of HardDec with $\beta=0.5$. In this evaluation, there

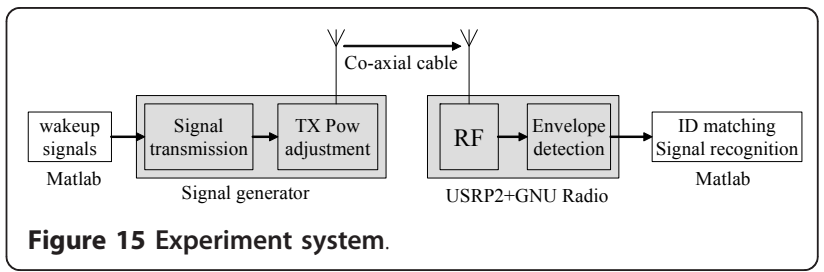


is no out-of-band interference. According to the FER results shown in Figure 16, HardDec at 100 kbps suffices at RSSI $=-82 \mathrm{dBm}$ when there is no out-of-band interference. But as discussed before, its performance is greatly degraded when interference does occur due to an imperfect BPF, as shown in Figure 9. SoftDec with merely 2 bits $\mathrm{A} / \mathrm{D}$ has a low FER, and provides about 3 to $4 \mathrm{~dB}$ SNR gain compared with HardDec (FER $=10^{-2}$ ).

\subsection{Review of power budget}

In this section, we review the power budget of the proposed wake-up receiver. The wake-up receiver has a simple structure: the RF part consists of a passive RF $\mathrm{BPF}$, an amplifier and an envelope detector; the baseband part consists of an A/D converter, a signal recognition module and a signal detection module. The amplifier and envelope detector of the RF part usually consume much power. But according to the results in the literatures [6-10], power consumption of the whole wake-up receiver (including the amplifier and envelope detector) for sensor networks is typically below $100 \mu \mathrm{W}$. As for the baseband part, 2-bit A/D is sufficient; the signal recognition module merely requires several comparators and a counter, and the signal detection module is only run when a signal is recognized as a wake-up signal. Accordingly, the baseband part can be realized by an ultra-low power micro-controller ${ }^{\mathrm{b}}$ whose maximal power consumption is no more than $0.9 \mathrm{~mW}$. This power consumption can be further reduced by using special hardware. Therefore, the conservative power budget of $1 \mathrm{~mW}$, discussed in Section 2.4, can be realized easily.

\section{Conclusion and future work}

We suggested wake-up transceivers for realizing ROD wireless LANs. As for the three key functions-signal co-

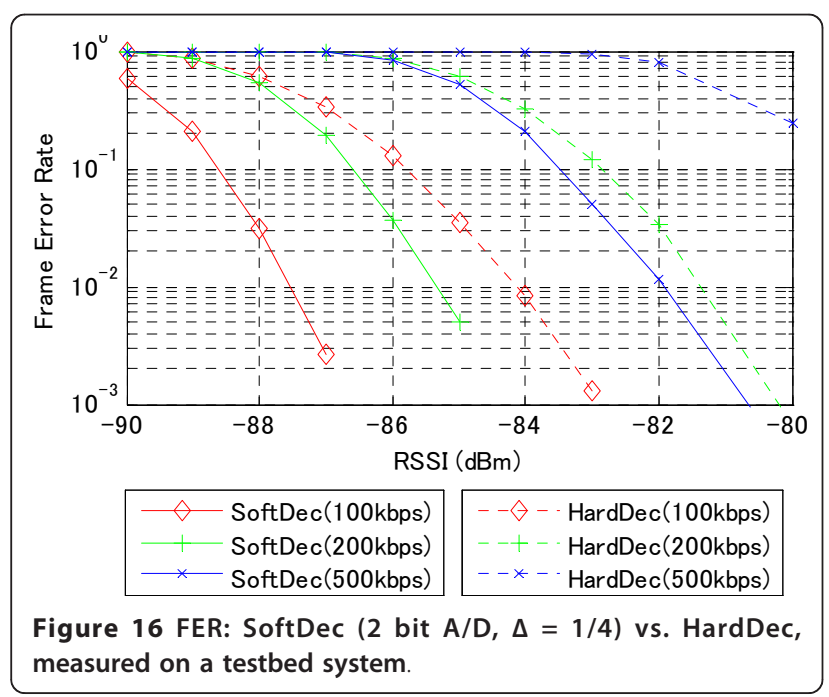

existence, signal detection and signal recognition, the first one is already solved in our previous work [18]. The performance of the latter two is much affected by the adjacent channel interference under imperfect BPFs. In this article, we examined the effect of imperfect RF BPF, and suggested SoftDec and optimal parameters for both signal detection and signal recognition. Simulation and experimental results confirm that the proposed method, with a low complexity, works well in the presence of moderate interferences. In the future, we will build the complete system and evaluate power consumption of the wake-up receiver as well as the whole system of an AP.

\section{Endnotes}

${ }^{\text {a }}$ Since each WID is transmitted in a single frame, FER has the same meaning as message error rate. ${ }^{\mathrm{b}}$ For example, MSP430, the 16-bit ultra-low power RISC mixed signal microprocessor from TI, consumes $300 \mu \mathrm{A} @ 3 \mathrm{~V}$ at the active mode and $0.6 \mu \mathrm{A} @ 3 \mathrm{~V}$ at the standby mode.

\section{Acknowledgements}

This work is supported by the Promotion program for Reducing Environmental loaD through ICT innovation (PREDICT) funded by Ministry of Internal Affairs and Communications, Japan.

\section{Author details}

'ATR Adaptive Communications Research Laboratories, 2-2-2 Hikaridai, Seikacho, Soraku-gun, Kyoto 619-0288, Japan ${ }^{2}$ Faculty of Engineering Science, Kansai University, 3-3-35 Yamate-cho, Suita, Osaka 564-8680, Japan

\section{Competing interests}

The authors are applying for a patent relating to the content of the manuscript.

Received: 31 August 2011 Accepted: 9 February 2012 Published: 9 February 2012

\section{References}

1. IC Wi-Fi, Shipments Forecast to Surpass 770 Million Units in 2010, http:// www.abiresearch.com/

2. R Bolla, R Bruschi, F Davoli, F Cucchietti, Energy efficiency in the future Internet: a survey of existing approaches and trends in energy-aware fixed network infrastructures. IEEE Commun Surv. Tutor. 13(2), 223-244 (2011)

3. N Mishra, K Chebrolu, B Raman, A Pathak, Wake-on-WLAN, in Proceedings of ACM WWW'06, Edinburgh, Scotland 761-769 (May 2006

4. AP Jardosh, K Papagiannaki, EM Belding, KC Almeroth, G lannaccone, B Vinnakota, Green WLANs: on-demand WLAN infrastructures. Mobile Netw Appl. 14(6), 798-814 (2009). doi:10.1007/s11036-008-0123-8

5. C Schurgers, V Tsiatsis, S Ganeriwal, M Srivastava, Topology management for sensor networks: exploiting latency and density, in Proceedings of ACM MobiHoc'02, Lausanne, Switzerland, 135-145 (June 2002)

6. JM Rabaey, J Ammer, T Karalar, S Li, B Otis, M Sheets, T Tuan, PicoRadios for wireless sensor networks: the next challenge in ultra-low power design. Proceedings of IEEE ISSCC'O2, San Francisco, USA, 200-201, 1, (February 2002)

7. DC Daly, AP Chandrakasan, An energy-efficient OOK transceiver for wireless sensor networks. IEEE J Solid State Circ. 42(5), 1003-1011 (2007)

8. X Huang, S Rampu, X Wang, G Dolmans, HD Groot, A 2.4GHz/915MHz 51 $\mu \mathrm{W}$ wake-up receiver with offset and noise suppression, in Proceedings of IEEE ISSCC'10, San Francisco, USA, 222-223, (February 2010)

9. B Otis, YH Chee, J Rabaey, A $400 \mu \mathrm{W}$ Rx, 1.6 mW Tx super-regenerative transceiver for wireless sensor networks, in Proceedings of IEEE ISSCC'05, San Francisco, USA, 396-397, (February 2005) 
10. NM Pletcher, S Gambini, J Rabaey, A $52 \mu \mathrm{W}$ wake-up receiver with $72 \mathrm{dBm}$ sensitivity using an uncertain-IF architecture. IEEE J Solid State Circ. 44(1), 269-280 (2009)

11. E Shih, P Bahl, MJ Sinclair, Wake on wireless: an event driven energy saving strategy for battery operated devices, in Proceedings of ACM MobiCom'02, Atlanta, USA, 160-171, (September 2002)

12. T Jin, G Noubir, B Sheng, WiZi-Cloud: application-transparent dual ZigbeeWiFi radios for low power Internet access, in Proceedings of IEEE INFOCOM'11, Shanghai, China, 1593-1601, (April 2011)

13. S Ishida, T Takiguchi, S Saruwatari, M Minami, H Morikawa, Evaluation of a wake-up wireless module with bloom-filter-based ID matching, in Proceedings of APSITT'10, Kuching, Malaysia, 1-6, (June 2010)

14. F Zhang, TD Todd, D Zhao, V Kezys, Power saving access points for IEEE 802.11 wireless network infrastructure. IEEE Trans Mobile Comput. 5(2), 144-156 (2006)

15. FR Dogar, P Steenkiste, K Papagiannaki, Catnap: exploiting high bandwidth wireless interfaces to save energy for mobile devices, in Proceedings of ACM MobiSys'10, San Francisco, USA, 107-122, (June 2010)

16. AMD white paper on WOL, http://www.networking.ibm.com/eji/ejiwake. html

17. Wake on Wireless LAN, http://revolutionwifi.blogspot.com/2010/11/wake-onwireless-lan.html

18. S Tang, $\mathrm{H}$ Yomo, Y Kondo, S Obana, Wakeup receiver for radio-on-demand wireless LANs, in Proceedings of IEEE Globecom'11, Houston, USA, (December, 2011)

19. I Demirkol, C Ersoy, E Onur, Wake-up receivers for wireless sensor networks: benefits and challenges. IEEE Wirel Commun. 16(4), 88-96 (2009)

20. J Lansford, A Stephens, R Nevo, Wi-Fi (802.11b) and Bluetooth: enabling coexistence. IEEE Netw. 15(5), 20-27 (2001). doi:10.1109/65.953230

21. EY Lin, J Rabaey, A Wolisz, Power-efficient rendezvous schemes for dense wireless sensor networks, Proceedings of IEEE ICC'04, Paris, France, 7, 3769-3776 (June 2004)

22. IEEE, Wireless LAN Medium Access Control (MAC) and Physical Layer (PHY) Specification (Piscataway, NJ, 2007)

23. IEEE, Carrier Sense Multiple Access with Collision Detection (CSMA/CD) Access Method and Physical Layer Specifications (Piscataway, NJ, 2008)

24. IEEE, Wireless Medium Access Control (MAC) and Physical Layer (PHY) Specifications for Low-Rate Wireless Personal Area Networks (WPANs) (Piscataway, NJ, 2007)

25. FCC, Code of Federal Regulations (CFR), Title 47 Telecommunication, 15.247

26. MIC, Equipment Ordinance (EO) for Regulating Radio Equipment, 49.20

27. T Jiang, Y Wu, An overview: peak-to-average power ratio reduction techniques for OFDM signals. IEEE Trans Broadcast. 54(2), 257-268 (2008)

28. DP Bertsekas, JN Tsitsiklis, Introduction to Probability, (Athena Scientific, Massachusetts, 2002)

29. QualNet, http://www.scalable-networks.com/products/qualnet/

30. GnuRadio, http://gnuradio.org/redmine/wiki/gnuradio

\section{Submit your manuscript to a SpringerOpen ${ }^{\mathcal{O}}$ journal and benefit from:}

- Convenient online submission

- Rigorous peer review

- Immediate publication on acceptance

- Open access: articles freely available online

- High visibility within the field

- Retaining the copyright to your article

Submit your next manuscript at $\gg$ springeropen.com 